\title{
Suizidprävention: Vorgehensweisen und Wirksamkeit
}

\author{
David Althaus \\ Klinik für Psychiatrie und Psychotherapie der Ludwig-Maximilians-Universität München, Deutschland
}

Schlüsselwörter

Suizid · Epidemiologie · Prävention, Suizid · Evaluation

\section{Zusammenfassung}

Laut offiziellen Statistiken nehmen sich in Deutschland jährlich 11000 Menschen das Leben. Noch vor gut 20 Jahren wurden jährlich fast 19000 Suizide registriert. Was sind die Ursachen für diesen Rückgang? Welche Rolle spielen suizidpräventive Maßnahmen in diesem Kontext? Verschiedene universelle Präventionsstrategien (z.B. erschwerter Zugang zu Suizidmethoden) wie auch selektive Herangehensweisen (Programme für spezielle Hochrisikogruppen; z.B. Patienten nach Suizidversuch) werden vorgestellt und in ihrer Wirksamkeit diskutiert. Ein streng wissenschaftlicher Nachweis der Wirksamkeit suizidpräventiver Maßnahmen gelingt dabei in den wenigsten Fällen. Weder die Rolle psychosozialer Interventionen noch die von Psychopharmaka lässt sich befriedigend quantifizieren. Vor allem methodische Probleme (kleine Fallzahlen, fehlende Randomisierung) erschweren die Interpretation der Daten. Die Kombination verschiedener Herangehensweisen dürfte sich zur Suizidprävention langfristig am besten eignen.

\section{Die Entwicklung der Suizidrate in Deutschland}

Laut Schätzungen der WHO nehmen sich jährlich weltweit rund 1 Million Menschen das Leben [Bertolote, 2001]. Damit sterben durch Suizid mehr Menschen als durch Unfälle, Kriege und Drogen. Die Notwendigkeit von Suizidprävention ist unbestritten und ist in den vergangenen Jahren verstärkt in den Fokus gerückt. Zahlreiche nationale wie regionale Programme zur Reduktion der Suizidraten wurden gestartet und viele Initi-

\section{Key Words}

Suicide $\cdot$ Epidemiology $\cdot$ Prevention, suicide $\cdot$ Evaluation

\section{Summary}

Suicide Prevention: Approaches and Evaluation

According to official statistics every year 11,000 persons in Germany die from suicide. 20 years ago nearly 19,000 suicides were registered. What are the causes for this decrease? Do suicide preventive measures contribute to the reduction of suicide rates? Different universal prevention strategies (e.g. restriction of access to means) and selective approaches (programs for special high-risk groups; e.g. patients after attempted suicide) are presented and discussed regarding their preventive value. In most cases it is hardly possible to scientifically prove the efficacy of suicide prevention strategies. Neither the role of psychosocial interventions nor the impact of psychotropic agents can be sufficiently quantified. Due to various methodological reasons (e.g. small sample sizes and the lack of randomization), interpretation of the data is difficult. In terms of a comprehensive approach of suicide prevention a combination of different activities should be most adequate in the long run.

ativen wurden gegründet, um Suizidprävention aktiv umzusetzen. Auf der anderen Seite stellt sich die Frage, inwieweit eine Reduktion der Suizidraten erreichbar ist und wie erfolgreich sich einzelne Maßnahmen auswirken können. Die Abnahme der amtlichen Suizidraten in Deutschland und in einigen anderen europäischen Ländern (unter anderem in Skandinavien) im Laufe der letzten zwei Jahrzehnte gibt zumindest einen Hinweis auf die Möglichkeit von Suizidprävention. Abbildung 1 zeigt die Suizidraten in Deutschland von 1982 bis 2002.

\begin{tabular}{|c|c|c|}
\hline KARGER & (c) 2005 S. Karger GmbH, Freiburg & $\begin{array}{l}\text { Dipl.-Psych. Dr. David Althaus } \\
\text { «Bündnis gegen Depression»e. V. }\end{array}$ \\
\hline $\begin{array}{l}\text { Fax +497614520714 } \\
\text { E-mail Information@Karger.de } \\
\text { www.karger.com }\end{array}$ & $\begin{array}{l}\text { Accessible online at: } \\
\text { www.karger.com/ver }\end{array}$ & $\begin{array}{l}\text { Psychiatrische Klinik der LMU München } \\
\text { Nussbaumstr. 7, 80336 München, Deutschland } \\
\text { Tel.+49 89 5160-5754, Fax -5557 } \\
\text { E-mail david.althaus@psy.med.uni-muenchen.de }\end{array}$ \\
\hline
\end{tabular}




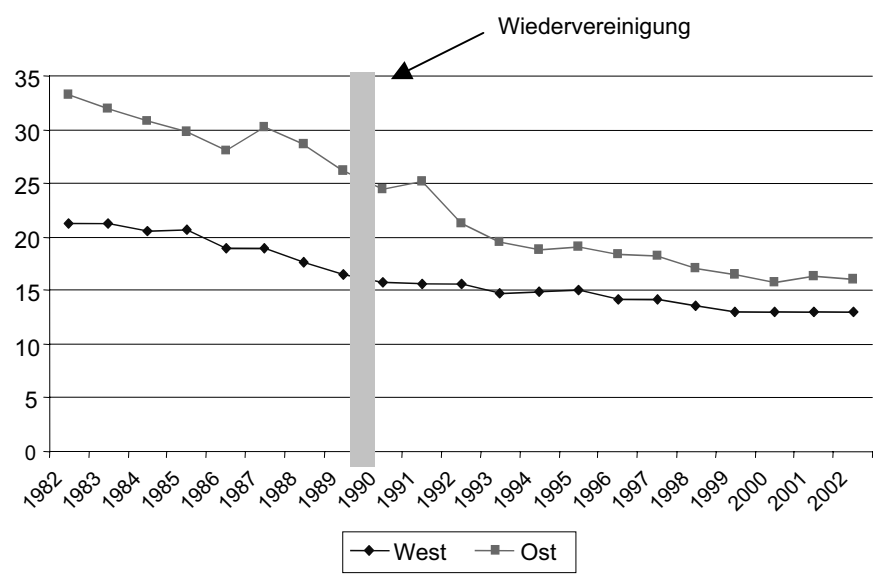

Abb. 1. Die Suizidraten je 100000 Einwohner in Ost- und Westdeutschland seit 1982.

Die jährliche Zahl der Suizide ist seit 1981 von knapp 19000 Fällen auf rund 11000 gesunken [Statistisches Bundesamt Deutschland, 2003]. Man muss bei der Interpretation dieser Veränderungen allerdings vorsichtig sein. Laut Schätzungen gibt es jährlich in Deutschland 170000 Fehlklassifikationen der Todesart [Madea und Dettmeyer, 2003]. Hinzu kommen jährlich rund 15000 Todesfälle mit unklarer Todesursache. Auch wenn in der ganz großen Mehrzahl dieser Fälle keine Selbsttötung vorliegt, besteht doch eine gewisse Unschärfe bei der Erfassung von Suiziden. Auch dürfte unter den Drogen- und Verkehrstoten ein gewisser Anteil an Suiziden sein. Der Rückgang amtlich verfügter Obduktionen in den vergangenen Jahren dürfte die Validität offizieller Statistiken zudem nicht verbessert haben (1980: 14,6\%, 1995: 1,2\%; [Deutsches Ärzteblatt, 2000]). Dennoch gibt es einige Hinweise, die sehr dafür sprechen, dass tatsächlich ein Rückgang der Suizidraten stattgefunden hat. Betrachtet man gezielt die Häufigkeit von Suizidmethoden [Statistisches Bundesamt Deutschland, 2003], bei denen kaum die Möglichkeit besteht, einen Suizid zu übersehen oder mit einem Unfall zu verwechseln, dann hat man einen recht validen Indikator für die tatsächliche Entwicklung. Insofern ist die Reduktion von Selbsttötungen durch Erhängen oder Strangulation von fast 8900 Fällen auf 5600 ein ermutigendes Indiz für einen Rückgang der Suizidraten. Mit großer Wahrscheinlichkeit ist daher davon auszugehen, dass in den vergangenen 25 Jahren tatsächlich ein substantieller Rückgang der Suizide um mehrere tausend Fälle pro Jahr stattfand. Doch nicht nur die Gesamtzahl der Suizide hat abgenommen, sondern auch die Suizidraten in den verschiedenen Altersgruppen. Eindrucksvoll zeigt sich das beispielsweise für Kinder und Jugendliche, deren Suizidrate sich mehr als halbiert hat (Abb. 2). Doch auch in den anderen Altersgruppen zeigen sich deutliche Rückgänge, so dass die gesunkenen Suizidraten insgesamt ein konsistenter Befund sind. Was genau sind die Ursachen für diese Entwicklung? Welche Veränderungen haben sich als suizidpräventiv erwiesen?

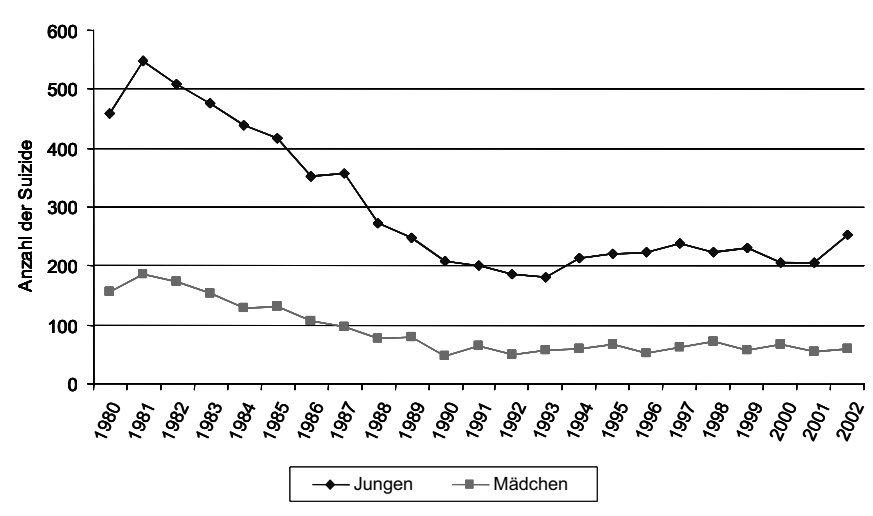

Abb. 2. Suizide von Jugendlichen in Deutschland seit 1980.

\section{Strategien innerhalb der Suizidprävention}

Grob gesprochen lassen sich innerhalb der Suizidprävention zwei wesentliche Herangehensweisen unterscheiden [Lewis et al., 1997]: Zum einen Strategien, die sich auf die gesamte Bevölkerung beziehen (universelle Strategien), zum andern eine Vorgehensweise, die sich auf bestimmte Teilgruppen oder Risikogruppen bezieht (selektive und indizierte Prävention). Universelle Prävention ist vor allem hilfreich, wenn es nicht ohne weiteres gelingt, Risikogruppen entweder klar zu identifizieren oder für spezifische Präventionsmaßnahmen zu gewinnen. Unter gesundheitsökonomischen Gesichtspunkten ist die generelle Gurtpflicht im Auto eine universelle primärpräventive Maßnahme zur Vorbeugung von schweren Unfallverletzungen. Sie trifft alle Autofahrer (und damit praktisch die gesamte Bevölkerung) und nicht nur diejenigen, die viel fahren oder einen besonders riskanten Fahrstil haben. Selektive Prävention ist im Gegensatz zu universeller vor allem dann sinnvoll, wenn klare Teil- und Risikogruppen beschreibbar sind, für die spezielle Interventionen entwickelt werden können. Schulische Anti-Drogen-Programme für Jugendliche sind ein Beispiel dafür.

Im Rahmen der Suizidprävention kommen universelle, selektive und indizierte Strategien zum Einsatz. Zum einen wurde wiederholt gezeigt, dass Menschen mit psychischen Erkrankungen eine besondere Risikogruppe für Suizid sind. Dementsprechend wurden für Menschen mit affektiven Störungen, Psychosen, Suchterkrankungen und Persönlichkeitsstörungen spezielle Interventionen und Programme ins Leben gerufen. Auf der anderen Seite gibt es aber auch Bemühungen, mit universellen Maßnahmen die Suizidraten zu beeinflussen. Ein typisches Beispiel dafür ist die Restriktion des Zugangs zu gefährlichen Suizidmethoden.

Anliegen dieser Arbeit ist es, einige wichtige suizidpräventive Ansätze darzustellen und zu diskutieren, inwieweit deren Wirksamkeit bislang wissenschaftlich belegt werden konnte [Althaus und Hegerl, 2003]. 


\section{Die Optimierung der Versorgung psychisch kranker Menschen}

In aufwendigen wissenschaftlichen Untersuchungen wurden bei Hunderten von Suiziden retrospektiv die Umstände untersucht, die zur Selbsttötung geführt haben. Diese so genannten «psychologischen Autopsiestudien» [Hawton et al., 1998a] zeigen, dass bei rund $90 \%$ aller Suizide eine psychiatrische Erkrankung im Vorfeld nachweisbar war. In rund 40-70\% aller Fälle waren dies depressive Erkrankungen [Lönnqvist, 2000], aber auch Suchterkrankungen und Psychosen spielten eine wichtige Rolle. Dementsprechend müsste eine konsequente Behandlung der Grunderkrankung einen bedeutsamen Einfluss auf die Suizidrate haben. Die Befunde der suizidpräventiven Wirkung verschiedener psychosozialer und pharmakologischer Strategien sind allerdings nicht immer so eindeutig, wie man annehmen möchte.

\section{Psychotherapeutische und psychosoziale Strategien}

\section{Krisenintervention und spezifische Psychotherapien}

Ausgehend von der klinischen Erfahrung würde man annehmen, dass insbesondere Krisenintervention (mit psychologischen und pharmakologischen Komponenten) einen entscheidenden Beitrag zur Suizidprävention leistet [vgl. Dormann, 2005]. In der Regel besteht die Hauptstrategie bei akuter Suizidalität darin, Zeit zu gewinnen, bis die akute Gefahr abklingt, um dann anschließend eine weitere (ursachenbezogene) Behandlung einzuleiten. So einleuchtend dieses Vorgehen erscheint, so wenig empirisch harte Befunde gibt es für die Effektivität dieses Vorgehens. Hier sind allerdings in erster Linie methodische Gründe verantwortlich zu machen. Ein experimentelles Vorgehen, bei dem einer Gruppe gefährdeter Menschen Krisenintervention vorenthalten werden müsste, ist aus ethischen Gründen undenkbar. Insofern wird es innerhalb der Suizidologie auch langfristig sehr schwer sein, wissenschaftliche Evidenz für augenscheinlich sinnvolle und unverzichtbare Interventionen zu erbringen.

Studien, die explizit versucht haben, die suizidpräventive Wirksamkeit spezifischer psychotherapeutischer Ansätze zu evaluieren, gibt es ebenfalls nur wenige. Zwar gibt es eine Vielzahl von Untersuchungen [Hawton et al., 2000], die sich mit der Frage beschäftigt haben, inwieweit psychologische Interventionen Einfluss auf die Wiederholungshäufigkeit von selbstverletzendem Verhalten haben («deliberate self-harm»). So wichtig und interessant diese Studien sind, so problematisch ist jedoch ihre Auswertung hinsichtlich einer potentiellen suizidpräventiven Wirkung. Gerade im Rahmen von Borderline-Persönlichkeitsstörungen finden habituelle Selbstverletzungen statt, die sich motivational deutlich von Suizidversuchen unterscheiden. In manchen Studien werden die Phänomene jedoch nicht immer klar von Suizidversuchen im engeren Sinn abgegrenzt. Ermutigende Hinweise gibt eine kontrollierte randomisierte
Untersuchung $(\mathrm{n}=44)$ von Shearin and Linnehan [1994] zur Wirksamkeit dialektisch-behavioraler Therapie (DBT). Patienten der Experimentalgruppe zeigten im Vergleich zur Kontrollgruppe (Routinebehandlung) während der DBT und im 6-Monats-Follow-up weniger Suizidversuche. Im 12-Monats-FollowUp konnten allerdings keine Unterschiede mehr zwischen Experimental- und Kontrollgruppe festgestellt werden. In einer neueren Studie [Verheul et al., 2003] ( $\mathrm{n}=58)$ zeigten sich während einer 52-wöchigen DBT-Behandlung zwar weniger Selbstverletzungen als in der Kontrollgruppe; hinsichtlich der Häufigkeit von Suizidversuchen unterschieden sich die beiden Gruppen jedoch nicht signifikant. In einer Studie von Salkowskis et al. [1990] zur Wirksamkeit von kognitiv-behavioraler Problemlösetherapie bei Patienten mit wiederholtem Suizidversuch $(\mathrm{n}=22)$ zeigte sich zwar eine vorübergehende Reduktion suizidaler Handlungen, allerdings konnte diese Studie bislang nicht repliziert werden. $\mathrm{Zu}$ fast allen vorliegenden Studien ist kritisch anzumerken, dass die untersuchten Stichproben sehr klein waren und Replikationen bislang ausstehen, so dass bezüglich einer suizidpräventiven Wirksamkeit kaum Schlussfolgerungen möglich sind. Eine aktuelle Literaturanalyse [Hepp et al., 2004] deutet eine Wirksamkeit psychosozialer Interventionen auf die Wiederholung von selbstverletzendem Verhalten an. Eine Reduktion von Suiziden konnte dagegen bisher nicht nachgewiesen werden.

\section{Krisendienst und Hilfe durch Laienhelfer}

Die Angebote für suizidgefährdete Menschen in Deutschland haben in den vergangenen Jahren deutlich zugenommen. Einen sehr schönen Überblick bietet die Homepage der Hamburger Beratungsstelle «neuland» (www.neuland.de). Ambulante Krisendienste, Krisentelefone und Telefonseelsorge bis hin zu Online-Beratung zeigen eine Vielfalt großer Unterstützungsangebote und bieten Hilfe an. Auf der anderen Seite ist wenig über die Effektivität derartiger Maßnahmen bekannt und ein direkter suizidpräventiver Einfluss kaum zu belegen. In England wurde beispielsweise untersucht, inwieweit die Samaritans (ehrenamtliche Laien, die in der Krisenintervention tätig sind) einen Beitrag zur Suizidprävention leisten. Die Befunde waren heterogen. Mal unterschieden sich Regionen mit entsprechenden Angeboten von solchen ohne Samaritans [Bagley, 1968], mal wurde kein Einfluss auf die Suizidrate festgestellt [Jennings et al., 1978]. Auch die Effizienz von Krisenzentren gilt als umstritten. In einer Analyse der Wirksamkeit von Suizidpräventionszentren in den USA fand Lester [1993] mittels multipler Regression immerhin einen konsistenten, wenn auch schwachen Einfluss auf die Suizidrate. Die Evaluation dieser Form von Hilfsangeboten scheint unter wissenschaftlicher Hinsicht besonders schwierig, weil sich hier eine reliable und valide Messung eindeutiger Erfolgskriterien als problematisch erweist.

\section{Suizidprävention durch Nachsorge und Kontaktangebote} Methodisch leichter ist dagegen die Evaluation spezifischer 
Interventionen bei einzelnen Risikogruppen. Dazu gehören insbesondere Menschen, die bereits einen Suizidversuch hinter sich haben. Motto et al. [1976, 1981] zeigten in einer randomisierten Studie, dass Patienten nach Suizidversuch dann seltener zu neuerlichen suizidalen Handlungen neigen, wenn sie in der Zeit nach dem Suizidversuch regelmäßig von der Klinik kontaktiert werden. Offensichtlich spielte dabei allein das Wissen um mögliche Hilfsangebote eine wesentliche Rolle. De Leo et al. [1995] konnten zeigen, dass ein so genannter «Telehelp-Service», bei dem 12135 ältere Menschen mit einem Sender ausgestattet wurden, über den sie notfalls Hilfe rufen konnten und über den sie regelmäßig kontaktiert wurden, um Dinge des täglichen Lebens zu klären, im Untersuchungszeitraum zu einer geringeren Suizidhäufigkeit führte, als dies - statistisch gesehen - für diese Stichprobe zu erwarten gewesen wäre (1 Suizid statt 7).

Morgan et al. [1993] randomisierten 212 Patienten nach Suizidversuch. Die eine Hälfte erhielt eine Notfallkarte, auf der eine Telefonnummer vermerkt war, wo sie im Falle einer erneuten suizidalen Krise anrufen sollten. Die Kontrollgruppe erhielt eine Routinebehandlung. Patienten, die mit der so genannten «Green-Card» ausgestattet waren, zeigten einen Trend zu weniger suizidalen Handlungen als jene der Kontrollgruppe ( $p=0,053)$. Eine Replikation mit einer größeren Stichprobe zeigte allerdings keine Gruppenunterschiede [Evans et al., 1999].

Gerade bei den psychosozialen Strategien zeigt sich, wie schwierig die Evaluation der suizidpräventiven Wirksamkeit ist. Zwar gehen viele Experten davon aus, dass die meisten Maßnahmen sinnvoll sind, auf der anderen Seite verweisen sie aber auch auf die Notwendigkeit weiterer vertiefender Studien mit größeren Stichproben [Hawton et al., 1998b].

\section{Die suizidpräventive Wirksamkeit von Medikamenten}

Die Wirksamkeit antidepressiver Medikation zur Behandlung affektiver Störungen ist durch viele Studien eindeutig belegt. Da Depression die am häufigsten mit Suizid assoziierte psychiatrische Störung ist, wäre aus theoretischen Gründen anzunehmen, dass antidepressive Medikation sich auch suizidpräventiv auswirkt. Auch wenn der klinische Eindruck und die praktische Erfahrung klar für einen antisuizidalen Effekt von Antidepressiva sprechen, liegen kaum gesicherte empirische Befunde vor, die einen direkten Einfluss auf die Häufigkeit suizidaler Handlungen nachweisen. Eine Reihe von Untersuchungen zeigt zwar eine Reduktion von Suizidgedanken [Beasley et al., 1991]. Fokussiert man jedoch auf eine substantielle Reduzierung der Suizidhäufigkeit und beschränkt sich auf Studien, die im Rahmen von prospektiven soliden Studiendesigns durchgeführt wurden, dann muss man feststellen, dass die Evidenzlage der suizidpräventiven Wirksamkeit von Antidepressiva unbefriedigend ist [Althaus und Hegerl, 2003]. Ein suizidfördernder Einfluss von SSRIs, der jüngst immer wieder diskutiert wurde, ist auf Basis vorliegender Daten nicht belegbar [Hegerl, 2004, 2005]. Auch über langfristige Auswirkungen liegen keine aussagekräftigen Daten vor, da die Beobachtungszeiträume in den Studien meist nur wenige Monate betrugen. Vor dem Hintergrund, dass über $50 \%$ der depressiven Patienten rezidivierende Krankheitsverläufe haben, bleibt daher die Frage offen, inwieweit Antidepressiva über Jahre hinweg die Suizidmortalität beeinflussen.

Günstiger sieht die Befundlage für Lithium aus [vgl. Pfennig et al., 2005], wenngleich auch hier ein Mangel an methodisch aussagekräftigen Studien vorliegt. Das größte Problem bei der Beurteilung der suizidpräventiven Wirksamkeit von Lithium besteht in den allgemein niedrigen Fallzahlen, der (meist) mangelnden Randomisierung und schwer kontrollierbaren Selektionseffekten. Ein Cochrane-Review [Burgess et al., 2001], das sich ausschließlich auf randomisierte kontrollierte Studien stützte, kam zum Ergebnis, dass es beim derzeitigen Forschungsstand noch nicht möglich sei, gültige Schlussfolgerungen über die Rolle von Lithiumtherapie innerhalb der Suizidprävention zu ziehen.

Noch immer ist die Anzahl der Studien, die sich mit suizidpräventiver Wirksamkeit von Neuroleptika befasst haben, relativ klein. Nach wie vor gilt die Arbeit von Meltzer und Okayli [1995] als ein wichtiger Hinweis für eine potentielle Wirksamkeit. In einem Prä-Post-Vergleich bei neuroleptikaresistenten Patienten mit Schizophrenie oder schizoaffektiven Störungen $(\mathrm{n}=88)$ zeigte sich unter Clozapin im Vergleich zum Zeitraum vor der Medikation eine niedrigere Suizidalität. Die Anzahl der besonders riskanten Suizidversuche sank von 5 auf 0 Fälle. Diese positiven Befunde werden von einer Untersuchung von Walker et al. [1997] unterstützt, bei der die Daten von 67072 mit Clozapin behandelte Patienten ausgewertet wurden. Dabei zeigte sich unter Clozapin ein deutlich geringeres Suizidrisiko $(-83 \%)$ als bei Patienten, die eine Clozapinbehandlung unterbrochen hatten. Kritisch anzumerken ist jedoch auch hier, dass es sich nicht um einen experimentellen Beleg der Wirksamkeit von atypischen Neuroleptika zur Suizidprävention handelt. Im Gegenteil lassen Daten einer Metaanalyse von Khan et al. [2001] Zweifel daran aufkommen. Ähnlich wie bei seinen Forschungen zur antisuizidalen Wirksamkeit von Antidepressiva untersuchte er Daten der Drug and Food Administration ( $\mathrm{n}=10$ 118). Weder Suizidraten noch Suizidversuchsraten unterschieden sich bei Personen, die Placebo, neuere oder ältere Antipsychotika erhalten hatten. Allerdings muss angemerkt werden, dass akute Suizidalität in der Regel ein Ausschlusskriterium für eine Studienteilnahme ist und daher auf dieser Datenbasis Schlussfolgerungen bzgl. Suizidprävention kaum möglich sind. Zusammenfassend kann gesagt werden, dass neuere Antipsychotika und Clozapin im Speziellen möglicherweise einen suizidpräventiven Effekt haben, allerdings sind auf der Basis der bisherigen Ergebnisse unspezifische Therapieeffekte und Selektionseffekte nicht auszuschließen, so dass hier weiterer Klärungsbedarf besteht. 


\section{Die Einschränkung verfügbarer Suizidmethoden}

Die Häufigkeit von Suizid wird auch dadurch beeinflusst, inwieweit zum Zeitpunkt einer suizidalen Krise sicher tödliche und einfach erreichbare Methoden zur Verfügung stehen. Wenn eine als unerträglich und hoffnungslos erlebte Situation sofort und sicher durch eine vermeintlich schmerzfreie Methode beendet werden kann, dann steigt die Gefahr eines Suizids erheblich. Nicht zufällig haben Anästhesisten und andere Personengruppen mit direktem Zugang zu sicheren Suizidmethoden ein erhöhtes Suizidrisiko [Birmingham und Ward, 1985]. In den USA, wo der Zugang zu Handfeuerwaffen für zivile Bürger wesentlich einfacher ist als in Deutschland, finden über 50\% der Suizide durch Erschießen statt. Im Durchschnitt erschießen sich in den USA jährlich 2500 Jugendliche zwischen 14 und 20 Jahren (62\% aller Suizide in dieser Altersgruppe) [Webster et al., 2004]. In Deutschland starben im Jahr 2002 dagegen nur insgesamt 15 Jugendliche durch Erschießen. Das entspricht rund 4\% der Suizide in diesem Alter. Auffällig in der amerikanischen Studie war dabei, dass die Anzahl der Jugendsuizide durch Handfeuerwaffen abhängig von der Waffengesetzgebung des jeweiligen Bundesstaates war. Je leichter Jugendliche selbst Waffen erwerben und besitzen konnten, desto höher war die Suizidrate mit Handfeuerwaffen.

Der beeindruckendste Befund für den Einfluss der Verfügbarkeit von Methoden auf die Suizidrate ergab sich in den Sechziger Jahren durch die so genannte «Coal-Gas-Story» [Kreitman, 1976]. Dabei konnte gezeigt werden, dass nach der Entgiftung des Hausgases ein dramatischer Rückgang von Suiziden in Großbritannien zu verzeichnen war. Steht eine sichere und häufig verwendete Methode zum Suizid nicht mehr zur Verfügung, so scheint eine erhebliche Anzahl potentieller Suizidenten nicht einfach eine alternative Methode zu wählen. Offenbar gibt es bei unterschiedlichen Personengruppen unterschiedliche Präferenzen, die nicht beliebig austauschbar sind. In dem Maße, wie Medikamente sicherer werden, hohe Gebäude eingezäunt werden, der Zugang zu Trassen von Hochgeschwindigkeitszügen schwieriger wird, sind auch Effekte auf die Suizidrate zu vermuten. Auch in Deutschland hat die Hausgasentgiftung zum Rückgang der Suizidrate beigetragen. Vor allem der drastische Rückgang der Suizide in den neuen Bundesländern seit der Wiedervereinigung von 3800 Fällen im Jahr 1992 auf 2200 Suizide im Jahr 2002 lässt sich zu rund einem Drittel der Entgiftung von Hausgas zuordnen (Abb. 3).

\section{Die Berichterstattung über Suizid in den Medien}

Nach Veröffentlichung von Goethes Sturm-und-Drang-Roman «Die Leiden des jungen Werther» kam es zu einer auffälligen Häufung von Suiziden, die explizit Bezug auf die Romanfigur nahmen [Ziegler und Hegerl, 2002]. Junge Männer kleideten sich ähnlich der Abbildung des Werther im Buch mit blauer

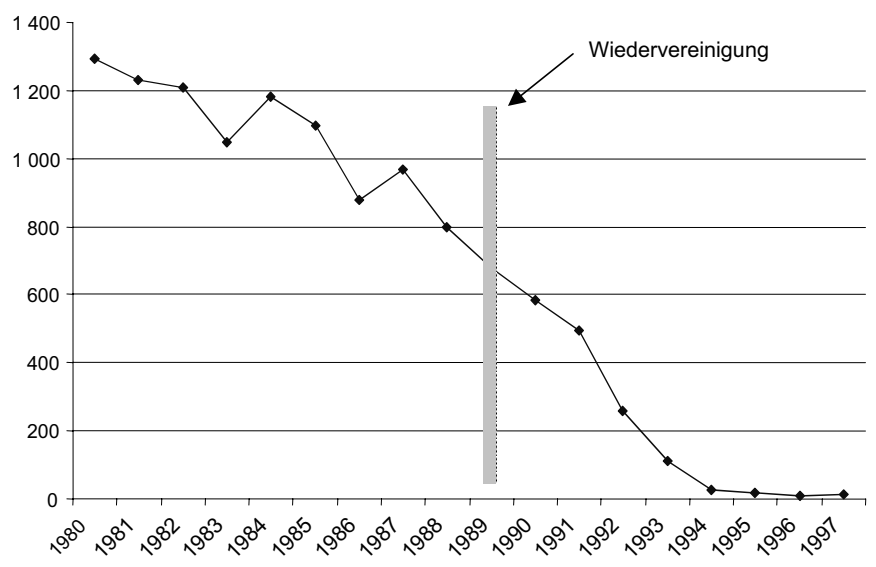

Abb. 3. Rückgang der Suizide durch Vergiftung mit Hausgas in Ostdeutschland. Seit ca. 1985 zeigt sich hier ein kontinuierlicher Rückgang. Dieser Trend setzt sich nach der sukzessiven Entgiftung des Hausgases ab Beginn der 90er Jahre fort.

Jacke und gelber Weste und töteten sich entsprechend des Vorbildes durch einen Kopfschuss. Diese als «Werther-Effekt» beschriebenen Imitationssuizide erschreckten nicht nur Goethe selbst, sondern führten auch dazu, dass das Buch vielerorts lange Zeit auf den Index gesetzt und verboten wurde. Erst rund 200 Jahre später fand erstmals eine systematische wissenschaftliche Analyse von Imitationssuiziden statt. Philips [1974] konnte zeigen, dass die in den Medien ausführlich beschriebenen Suizide prominenter Personen mit den Suiziden in der Allgemeinbevölkerung korrelierten. Inzwischen gilt es als unbestritten, dass ungünstige Modelle einen direkten Einfluss auf latent gefährdete Menschen haben und Suizide auslösen können. So zeigte sich, dass nach Ausstrahlung einer Fernsehserie («Tod eines Schülers»), in dem wiederholt der Eisenbahnsuizid eines jungen Mannes gezeigt wurde, die Suizidrate innerhalb der entsprechenden Zielgruppe (junge Männer) mit der identischen Methode (Eisenbahnsuizid) in einem Zeitfenster von mehreren Wochen signifikant zunahm [Schmidtke und Häfner, 1988]. Bei einer Zweitausstrahlung der Sendung Jahre später durch einen Privatsender zeigte sich ein ähnlicher Effekt, so dass hier im Rahmen eines Test-Retest-Designs der mediale Einfluss auf Suizidalität zweifelsfrei nachgewiesen wurde. Weit seltener sind dagegen Untersuchungen, die zeigen, dass durch eine Veränderung der Berichterstattung über Suizid Selbsttötungen verhindert werden können [Sonneck et al., 1994]. In Wien war es in den Jahren 1985-1987 zu einem deutlichen Anstieg von U-Bahn-Suiziden gekommen, nachdem über dieses Thema ausführlich und dramatisch in den Massenblättern berichtet wurde. Nachdem mit den entsprechenden Zeitungen eine Vereinbarung getroffen wurde, zukünftig nicht mehr über U-Bahn-Suizide zu berichten, nahm die Häufigkeit dieser Methode signifikant ab. Aus wissenschaftlicher Sicht ist es dennoch schwer abzuschätzen, wie stark der Einfluss negativer Modelle auf suizidales Verhalten tatsächlich ist. Insbesondere die Frage, ob möglicherweise nicht nur eine Verschiebung 


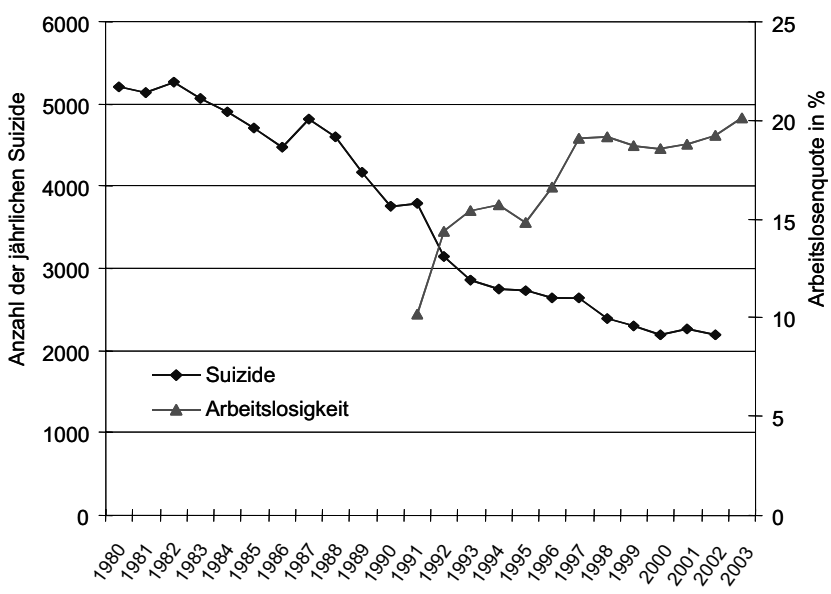

Abb. 4. Anzahl jährlicher Suizide und Arbeitslosenrate in Ostdeutschland seit der Wiedervereinigung (1990).

des Suizidzeitpunkts und der Methodenwahl stattfindet, lässt sich nicht befriedigend klären.

\section{Der Einfluss gesellschaftlicher Rahmenbedingungen auf Suizidalität}

Welchen Einfluss haben gesellschaftliche und ökonomische Rahmenbedingungen auf die Variationen der Suizidraten? Wie würde sich beispielsweise eine Reduktion der Arbeitslosigkeit auswirken? Durkheim [1973] hat sich bereits Ende des 19. Jahrhunderts in einer Zeit epochaler gesellschaftlicher Veränderungen aus soziologischer Perspektive mit dem Phänomen Suizid auseinander gesetzt. Die Ursachen des Suizids sind nach seiner Analyse vor allem im Verhältnis zwischen Individuum und Gesellschaft zu sehen. Laut Durkheim hängt die Höhe der Suizidrate vom Grad der sozialen Integration einer Gesellschaft ab. Die Suizidrate ist dann hoch, wenn die soziale Integration in einer Gesellschaft niedrig ist. Je weniger ein Mensch an die Normen und Wertvorstellungen der Gesellschaft gebunden ist, desto höher ist sein Suizidrisiko. Laut Durkheim ist dann die Suizidrate am niedrigsten, wenn die freie Entfaltung des einzelnen und die Gebundenheit an das kollektive Wertesystem im Gleichgewicht sind. In diesem Sinne sieht er die Suizidraten auch stark abhängig vom Grad der «Anomie» innerhalb einer Gesellschaft. Damit ist der Mangel an klarer Normsetzung und gesellschaftlicher Kontrolle gemeint, der letztendlich mit einen Verlust an Solidarität und sozialer Bindung einhergeht. Durkheim sieht vor allem in den Lebensbedingungen moderner Gesellschaften, die mit Werteverfall und zunehmender Individualisierung bei höherem persönlichen Wohlstand einhergehen, einen der Hauptgründe steigender Suizidraten.

Die Theorie Durkheims ist auf den ersten Blick in mancher Hinsicht einleuchtend, allerdings ist die Gültigkeit seiner The- sen bis heute nicht belegt. Es gelingt kaum, die konkreten soziologischen Einflussfaktoren dingfest zu machen und empirisch zu untersuchen. Welche gesellschaftlichen Faktoren könnten in den vergangenen 20 Jahren in Deutschland zu einem Rückgang der Suizidrate geführt haben? Gab es nicht eher kritische Entwicklungen? Der Anteil der alten Menschen (einer Hauptrisikogruppe für Suizid) nimmt kontinuierlich zu, der Arbeitsmarkt fordert stärker denn je Mobilität und Flexibilität, familiäre Strukturen verlieren dagegen mehr und mehr an Bedeutung. Die zunehmende Vereinzelung und Individualisierung, der starke Anstieg der Arbeitslosigkeit, die immer deutlichere Schere zwischen Arm und Reich sind Faktoren, die nach Durkheim mit steigenden Suizidraten verbunden sein müssten. Tatsächlich aber scheinen gesellschaftliche Faktoren kaum je in linearer Verknüpfung zur Suizidalität zu stehen. So ist zwar bekannt, dass Arbeitslosigkeit wie auch soziale Isolation zu den Auslösebedingungen von Suizid gehören können; eine kausale Verknüpfung ist indessen nicht belegbar. In einer dänischen Studie wurde gezeigt, dass Arbeitslosigkeit nur 3\% der Variation der Suizidraten erklärte [Mortensen et al., 2000]. In einer Analyse von deutschen Arbeitslosenstatistiken und Suizidraten wurden ebenfalls keine Zusammenhänge zwischen beiden Phänomenen gefunden [Häfner, 1998]. Die Entwicklung der Suizid- und Arbeitslosenraten in den neuen Bundesländern seit der Wiedervereinigung zeigt einen geradezu paradoxen Befund: Trotz der dort in den letzten Jahren dramatisch gewachsenen Massenarbeitslosigkeit nahmen die Suizidraten sehr deutlich ab (Abb. 4).

Gerade die Entwicklung in den neuen Ländern hätte laut Durkheim mit einem Anwachsen der Suizidrate verbunden sein müssen: größerer persönlicher materieller Wohlstand bei wachsender gesellschaftlicher Unsicherheit, Verlust an klaren gesellschaftlichen Normen und Regeln, Verlust von Kohärenz und Zusammengehörigkeitsgefühl etc. An diesem Beispiel wird deutlich, wie schwierig die Interpretation von Suizidraten vor dem Hintergrund gesellschaftlicher Rahmenbedingungen tatsächlich ist. Empirisch überprüfte Theorien sind dabei Mangelware.

\section{Diskussion}

Die in Deutschland kontinuierlich sinkende Suizidhäufigkeit seit den 80er Jahren von fast 19000 auf nunmehr rund 11000 Fälle pro Jahr gibt ermutigende Hinweise, dass Suizidprävention möglich ist. Auch wenn ein Teil der Reduktionen durch eine höhere Anzahl an Drogenopfern und ungeklärte Todesursachen kompensiert wird, kann davon ausgegangen werden, dass es in den beiden vergangenen Jahrzehnten tatsächlich eine Reduktion um mehrere tausend Suizide pro Jahr gegeben hat. Genaue Ursachen für diesen Rückgang zu benennen fällt schwer. Ein Grund dürfte darin liegen, dass einige früher weit verbreitete Suizidmethoden wie z.B. tödliches Hausgas oder hochtoxische Gifte aus der Landwirtschaft heute nicht mehr 
zur Verfügung stehen. Auch der Zugang zu gefährlichen Orten ist heute eingeschränkter als noch früher (Hausdächer, Brückengeländer etc.). Doch wie groß ist die Wirkung all dieser Maßnahmen? Wie wir gesehen haben, ist die Evaluation suizidpräventiver Maßnahmen (egal ob psychosozial oder medikamentös) insgesamt sehr schwierig. Suizid ist ein seltenes Ereignis, und bei der Untersuchung spezieller Interventionen innerhalb eines kontrollierten Designs sind sehr große Stichprobenumfänge notwendig, um zeigen zu können, dass ein Verfahren signifikant wirksamer ist als eine Placebobehandlung. In Fallzahlschätzungen [Gunnell und Frankel, 1994] kommt man zum Ergebnis, dass selbst bei der Hochrisikogruppe von Patienten nach Suizidversuch über 3000 Fälle eingeschlossen werden müssten, um eine $50 \%$ ige Reduktion der Suizidrate nachweisen zu können (bei 80\% Teststärke und 5\% Fehlerwahrscheinlichkeit). Aus diesen ernüchternden Zahlen darf die Schlussfolgerung gezogen werden, dass Studien zur Untersuchung suizidpräventiver Wirksamkeit in der Regel entweder von vornherein wegen viel zu geringer Teststärke (Power) zum Scheitern verurteilt sind oder ihre Organisation und Umsetzung extrem ressourcenintensiv wären. Dies ist einer der entscheidenden Gründe, warum innerhalb dieses Forschungsfeldes so wenig verlässliche Aussagen vorliegen. Insofern ist gerade bei der Untersuchung suizidpräventiver Interventionen $\mathrm{zu}$ fragen, inwiefern es unter wissenschaftlichen Gesichtspunkten überhaupt sinnvoll ist, bei der Evaluierung eines suizidpräventiven Effekts ein traditionelles experimentelles Forschungsparadigma anzuwenden, das auf Randomisierung, Kontrollgruppe und Irrtumswahrscheinlichkeit basiert.

Daneben gibt es jedoch auch erhebliche ethische Barrieren bei der Durchführung aussagekräftiger Studien. Beispielsweise gilt Lithium als eine Substanz, die mit sehr hoher Wahrscheinlichkeit einen spezifischen suizidpräventiven Effekt hat, auf der anderen Seite sind diese Erkenntnisse hauptsächlich in quasiexperimentellen Untersuchungen und Langzeitbeobachtungen gewonnen worden, und es liegen noch immer kaum Daten aus randomisierten kontrollierten Studien vor. Es ist ethisch kaum vertretbar, Hochrisikopatienten (z.B. mit rezidivierenden schweren depressiven Episoden) zur Suizidprophylaxe nach einem Zufallsprinzip entweder Lithium oder ein Placebo zu geben. Dies würde ja bedeuten, dass man der Hälfte der suizidgefährdeten Menschen per Los eine wahrscheinlich Suizid verhindernde Medikation nicht zukommen ließe.

Ein weiterer umstrittener Diskussionspunkt innerhalb der Suizidologie ist schließlich die Tragweite einzelner suizidpräventiver Verfahren. Auch wenn beispielsweise große Einigkeit darüber besteht, dass eine ungünstige Medienberichterstattung Imitationssuizide auslösen kann, so ist doch unklar, in welchem zahlenmäßigen Umfang sich dieser Faktor tatsächlich niederschlägt. Insbesondere suizidpräventive Maßnahmen, die für Hochrisikogruppen konzipiert wurden, kranken an dieser Unschärfe, da auch heute noch ein Großteil potentiell gefährdeter Menschen nicht mit Institutionen in Kontakt steht, die überhaupt derartige Maßnahmen einleiten könnten. Selbst wenn dialektisch-behaviorale Therapie eine suizidpräventive Wirkung hat, so ist zu fragen, in welchem Umfang dieses Verfahren zukünftig tatsächlich außerhalb von spezifischen, hoch spezialisierten Kliniken zum Einsatz kommen kann.

\section{Ausblick}

Welche Lehren sind für die wissenschaftliche Evaluation von Suizidprävention zu ziehen? Einerseits muss man eingestehen, dass viele der derzeit praktizierten nieder- und hochschwelligen Interventionen keinen eindeutigen Beleg ihrer Wirksamkeit erbringen können. Auf der anderen Seite erscheinen die Notwendigkeit und der Nutzen der verschiedenen Ansätze zumindest für einen eng umschriebenen Personenkreis sehr wahrscheinlich. Das Puzzle der Suizidprävention besteht aus vielen Einzelteilen. Zweifelsohne sind die mit Suizidalität verbundenen Ursachen, Auslöser und Prozesse so komplex, dass es sinnvoll ist, verschiedenste Ansätze in der Primär-, Sekundär- und Tertiärprävention zu verfolgen. Die Verbesserung psychiatrischer und psychotherapeutischer Versorgung in den vergangenen 25 Jahren dürfte bei den rückläufigen Suizidraten in Deutschland einen wichtigen Einfluss gehabt haben. Auf der anderen Seite wird es notwendig sein, auch universelle Präventionsstrategien weiterzuverfolgen, um eine größere Zielgruppe erreichen zu können. Im Rahmen eines depressions- und suizidpräventiven Mehrebenen-Programms in Nürnberg [Hegerl et al., 2003] konnte gezeigt werden, dass gerade die Kombination von verschiedenen Ansätzen Erfolg versprechend sein kann [vgl. Hegerl, 2005] Neben einer engen Kooperation mit Hausärzten und der Schulung verschiedener Multiplikatorengruppen (z.B. Lehrer, Pfarrer, Altenpflegekräfte, Polizei) stand vor allem die Aufklärung der Bevölkerung über Depression im Mittelpunkt der Aktivitäten. In einem kontrollierten Studiendesign konnte ein signifikanter Rückgang von Suizidversuchen (-26\%) nachgewiesen werden.

Die Deutsche Gesellschaft für Suizidprävention (DGS) hat 2001 eine Arbeitsgruppe gegründet, um in Zusammenarbeit mit allen relevanten gesellschaftlichen Gruppierungen und Vereinigungen ein nationales Suizidpräventionsprogramm zu initiieren. Die verschiedenen Arbeitsgruppen haben dabei die Aufgabe, einen konkreten Maßnahmenkatalog und Evaluationsstrategien zu erarbeiten, die dann in den nächsten Jahren umgesetzt werden sollen (www.suizidpraevention-deutschland.de).

\section{Dank}

Diese Arbeit entstand im Rahmen des Kompetenznetzes Depression, Suizidalität und wurde vom Bundesministerium für Bildung und Forschung (BMBF) unterstützt. 


\section{Literatur}

Althaus D, Hegerl U: The evaluation of suicide prevention activities: State of the art. World J Biol Psychiatry 2003;4:156-165.

Bagley C: The evaluation of a suicide prevention scheme by an ecological method. Soc Sci Med 1968;2: 114.

Beasley CM Jr, Dornseif BE, Bosomworth JC, Sayler ME, Rampey AH Jr, Heiligenstein JH, Thompson VL, Murphy DJ, Masica DN: Fluoxetine and suicide: A meta-analysis of controlled trials of treatment for depression BMJ 1991;303:685-692.

Bertolote JM: Suicide in the world: An epidemiological overview: 1959-2000; in Wasserman D (ed): Suicide - an Unnecessary Death. London, Matin Dunitz, 2001, pp 3-10.

Birmingham PK, Ward RJ: A high-risk suicide group: The anesthesiologist involved in litigation. Am J Psychiatry 1985;142:1225-1226.

Burgess S, Geddes JR, Hawton K, Townsend E, Jamison KR, Goodwin G: Lithium for maintenance treatment of mood disorders. Cochrane Database Syst Rev 2001;CD003013.

De Leo D, Carollo G, Dello Buono M: Lower suicide rates associated with a Tele-Help/Tele-Check service for the elderly at home. Am J Psychiatry 1995;152:632634.

Deutsches Ärzteblatt (Hrsg): Obduktionen: Bedenklicher Rückgang. Deutsches Ärztebl 2000;97:A-797.

Dormann W: Pro und Contra von Verträgen bei Patienten in akuten suizidalen Krisen. Verhaltenstherapie DOI: $10.159 / 000082736$.

Durkheim E: Der Selbstmord (deutsche Übersetzung von 1887). Luchterhand, 1973

Evans MO, Morgan HG, Hayward A, Gunnell DJ: Crisis telephone consultation for deliberate self-harm patients: Effects on repetition. Br J Psychiatry 1999;175: 23-27.

Gunnell D, Frankel S: Prevention of suicide: Aspirations and evidence. BMJ 1994;308:1227-1233.

Häfner H: Arbeitslosigkeit und Suizidalität. Suizidprophylaxe 1998;25:141-152.

Hawton K, Appleby L, Platt S, Foster T, Cooper J, Malmberg A, Simkin S: The psychological autopsy approach to studying suicide: A review of methodological issues. J Affect Disord 1998a;50:269-276.
Hawton K, Arensman E, Townsend E, Bremner S, Feldman E, Goldney RD, Gunnell D, Hazell P, van Heeringen K, House A, Owens D, Sakinofsky I, Traskman-Bendz L: Deliberate self harm: Systematic review of efficacy of psychosocial and pharmacological treatments in preventing repetition. BMJ 1998b;317: 441-447.

Hawton K, Townsend E, Arensman E, Gunnell D, Hazell P, House A, van Heeringen K: Psychosocial versus pharmacological treatments for deliberate self harm. Cochrane Database Syst Rev 2000;CD001764.

Hegerl U: Antidepressiva und Suizidalität. Verhaltenstherapie 2004;14:218-219.

Hegerl U: Depression und Suizidalität. Verhaltenstherapie DOI: $10.159 / 000083697$

Hegerl U, Althaus D, Niklewski G, Schmidtke A: Optimierte Versorgung depressiver Patienten und Suizidprävention: Ergebnisse des «Nürnberger Bündnisses gegen Depression». Deutsches Ärztebl 2003;100:27322737

Hepp U, Wittmann L, Schnyder U, Michel K: Psychological and psychosocial interventions after attempted suicide: An overview of treatment studies. Crisis 2004;25:108-117.

Jennings C, Barraclough BM, Moss JR: Have the Samaritans lowered the suicide rate? A controlled study. Psychol Med 1978;8:413-422.

Khan A, Khan SR, Leventhal RM, Brown WA: Symptom reduction and suicide risk among patients treated with placebo in antipsychotic clinical trials: An analysis of the food and drug administration database. Am J Psychiatry 2001;158:1449-1454.

Kreitmann N: The coal gas story. United Kingdom suicide rates, 1960-71. Br J Prev Soc Med 1976;30:86-93. Lester D: The effectiveness of suicide prevention centers. Suicide Life Threat Behav 1993;23:263-267.

Lewis G, Hawton K, Jones P: Strategies for preventing suicide. Br J Psychiatry 1997;171:351-354.

Lönnqvist J: Psychiatric aspects of suicidal behaviour: Depression; in Hawton K, van Heeringen K (eds): The International Handbook of Suicide and Attempted Suicide. John Wiley \& Sons, 2000.

Madea B, Dettmeyer R: Ärztliche Leichenschau und Todesbescheinigungen. Deutsches Ärztebl 2003;48: 3161-3179.

Meltzer HY, Okayli G: Reduction of suicidality during clozapine treatment of neuroleptic-resistant schizophrenia: Impact on risk-benefit assessment. Am J Psychiatry 1995;152:183-190.
Morgan HG, Jones EM, Owen JH: Secondary prevention of non-fatal deliberate self-harm. The green card study. Br J Psychiatry 1993;163:111-112.

Mortensen PB, Agerbo E, Erikson T, Qin P, Westergaard Nielsen N: Psychiatric illness and risk factors for suicide in Denmark. Lancet 2000;355:9-12.

Motto JA: Suicide prevention for high-risk persons who refuse treatment. Suicide Quart J Life Threat Behav 1976;6:223-230.

Motto JA, Heilbron DC, Juster RP, Bostrom AG: Communication as a Suicide Prevention Program; in Soubrier JP, Vedrinne J (eds): Dépression et suicide. Paris, Pergamon, 1981.

Pfennig A, Berghöfer A, Bauer M: Medikamentöse Behandlung der Suizidalität. Verhaltenstherapie DOI: 10.159/000083490.

Phillips DP: The influence of suggestion on suicide: Substantive and theroretical implications of the Werther effect. Am Sociol Rev 1974;39:340-354.

Salkovskis PM, Atha C, Storer D: Cognitive-behavioural problem solving in the treatment of patients who repeatedly attempt suicide. A controlled trial. $\mathrm{Br}$ J Psychiatry 1990;157:871-876.

Schmidtke A, Häfner H: The Werther effect after television films: New evidence for an old hypothesis. Psychol Med 1988;18:665-676.

Shearin EN, Linehan MM: Dialectical behavior therapy for borderline personality disorder: Theoretical and empirical foundations. Acta Psychiatr Scand Suppl 1994; 79:61-68.

Sonneck G, Etzersdorfer E, Nagel KS: Imitative suicide on the Viennese subway. Soc Sci Med 1994;38: 453-457.

Statistisches Bundesamt Deutschland (Hrsg): Todesursachenstatistik 1982-2002. 2003.

Verheul R, Van Den Bosch LM, Koeter MW, De Ridder MA, Stijnen T, Van Den Brink W: Dialectical behaviour therapy for women with borderline personality disorder: 12-month, randomised clinical trial in the Netherlands. Br J Psychiatry 2003;182:135-140.

Walker AM, Lanza LL, Arellano F, Rothman KJ Mortality in current and former users of clozapine. Epidemiology 1997;8:671-677.

Webster DW, Vernick JS, Zeoli AM, Manganello JA Association between youth-focused firearm laws and youth suicides. JAMA 2004;292:594-601.

Ziegler W, Hegerl U: Der Werthereffekt: Bedeutung, Mechanismen und Konsequenzen. Nervenarzt 2002;73: $41-49$ 